\title{
Unstable Electron Pairing and the Energy Loan Model of Enzyme Catalysis
}

\author{
M. ConRaD ${ }^{\dagger}$ \\ Department of Computer and Communication Sciences, \\ University of Michigan, Ann Arbor, Michigan 48104, U.S.A.
}

(Received 4 April 1978, and in revised form 28 December 1978)

$\Lambda$ theory of enzyme catalysis is described which utilizes a thermodynamically consistent construction of a free energy diagram with different pathways for complex formation and decomposition. The switch to the decomposition pathway occurs when downward uncertainty and thermal fluctuations make possible a short-lived potential energy dominance in which parallel spin electrons are paired and thus free to drop below the energy floor normally maintained by the Pauli exclusion principle. Such pairing is possible if van der Waal's and other weak interactions holding the complex together impose confinement constraints on parallel spin electrons, thereby both increasing uncertainty fluctuations in their kinetic energy and weakly favoring a phase correlation in their motion (which can be interpreted in terms of an exchange of virtual particles). The paired configuration is highly unstable and thus energy released by pair falling is either immediately recaptured to re-establish a normal orbital structure or, if the pair persists long enough to produce a nuclear motion, recaptured at the end of this motion. In the latter case the release of energy can be thought of as an energy loan which finances the switch to the lower activation energy pathway without compromising an energy-balanced regeneration of the enzyme. The advantage is that the complex (because of its instability) has a real free energy which is lower than the free energy which would bc assigned to it on the basis of its equilibrium concentration. This increases the specificity and speed of complex formation without decreasing the speed of decomposition. The theory predicts that the magnetic moment which marks the pair should accompany the nuclear (e.g. allosteric) motion and that the pair formation stage of the enzymatic process should have an anomalous temperature dependence. Variations of the model may be constructed to deal with a number of processes involving macromolecular motions, including sequential processes in catalysis, allosteric control, persistent molecular motions, self-assembly, energy transfer, channeled transport, and protection against inhibitors.

\section{Introduction}

Enzymes are remarkable not only for their strong effect on the rates of reactions, but also for their high specificity. This specificity is generally

$\dagger$ Present address: Departments of Computer Science and Biology, Wayne State University. Detroit, Michigan 48202, U.S.A.

$0022-5193 / 79 / 140137+20 \$ 02.00 / 0$ 
believed to have its basis in shape complementarity of enzyme and substrate along with the $r^{-6}$ dependence of the van der Waal's interactions (allowing for a strong, additive effect only in the event of close fitting). If the complex formed as a result of this close fit were stable, specificity would clearly preclude speed. In fact the shape of the enzyme is not a static property and so far as is known the catalytic process is always accompanied by a significant change in the nuclear configuration. An adequate theory of enzymatic catalysis must thus incorporate not only interactions responsible for specificity and bond alteration, but also interactions which account for the instability of the enzyme-substrate complex and for the nuclear (e.g. allosteric) motion.

In this paper a mechanism is proposed which produces a nuclear motion, hence a source of instability, and which has the interesting property that it does so on the basis of an interaction which has its origin in the same shape complementarity which gives rise to specificity. The basic idea is that strong van der Waal's and other weak interactions between complementary surfaces of enzyme and substrate impose confinement constraints on parallel spin clectrons, in effect creating an attractive interaction. Under conditions of downward thermal and quantum fluctuations (in kinetic energy) this attraction assumes a transient dominance, thereby establishing a short-lived electron pair which falls in the direction of a low energy orbit belonging to many nuclei, then breaks up and re-establishes a normal electronic structure. The importance of this short-lived destabilization of the electronic structure is that it provides an "energy loan" which makes possible a nuclear motion, thereby dynamically opening up an alternative reaction route which favors decomposition of the complex. The same mechanism is also capable of contributing to other biologically significant macromolecular motions as well.

Electron pairing (involving antiparallel spin electrons) is now known to be the basis of superconductivity and according to the well-known theory of Bardecn, Cooper \& Schrieffer (1957) it is the phonons associated with nuclear oscillations which are responsible for this pairing. The idea that a superconductive or superfluid state might lie at the basis of life phenomena was considered by early students of low temperature physics (Schrödinger, 1944; London, 1950). More recently Little (1964) has suggested the possibility of an organic superconductor based on electronic rather than nuclear vibrations, Pattee (1968) has discussed the possibility that the reliability of molecular biological processes involves a fundamental form of thermal isolation analogous to the superconductive state, Fröhlich (1968) has suggested that the coherent behavior of biological systems involves a Bose condensation of phonons associated with collective, dipole oscillations, 
and Josephson (personal communication) has considered the low temperature analogy in relation to mental processes. The present theory (Conrad, 1978) also draws on low temperature ideas to the extent that electron pairing plays the key role. However, the process under consideration is not a phase transition and the resulting behavior should not be identified with superconductivity or any form of persistent current or motion based on permanent pairing. On the contrary, what is important in catalysis is impermanence, hence unstable rather than stable forms of electronic structure.

\section{Conditions for Transient Pairing}

The generality of canonical methods requires simplifications which are inappropriate to the essential properties of complex systems such as enzymes. A constructive approach, involving direct use of basic physical principles to determine features of models which fulfill fundamental requirements, is more promising. The fundamental requirements, discussed in sections 6 and 7, are thermodynamic. They suggest transient pairing. Required for transient pairing is a coupling interaction between electrons (exceeding the screened Coulomb repulsion), together with suitable thermal and quantum fluctuations. Considering each of these in more detail:

\section{(A) CONSTRAINT-BASED COUPLING INTERACTION}

The source constraint is a cumulative van der Waal's attraction between complementary structures of enzyme and substrate which is sufficiently strong to push into close juxtaposition chemical moities which ordinarily repel because of nonbonding, parallel spin electrons (see Fig. 1). The cumulative, specific attraction may also be augmented by significant nonspecific wcak interactions (hydrogen bonding, hydrophobic interactions, ionic attractions, and others). If the electronic structure of the system is such that escape routes for these electrons are blocked, they will be forced together by the van der Waal's constraint while at the same time repelling because of the exclusion principle and to a much lesser extent because of a (screened) Coulomb repulsion. The net result is a spatial confinement of the electrons which favors the appearance of a phase correlation in their motion. Taking the point of view that the electrons are moving in a given nuclear potential, the coupling interaction could be thought of in terms of a compression of the average (minimum energy) distance between the constrained nuclei, with the consequence that electrons repel each other because of exclusion interference 

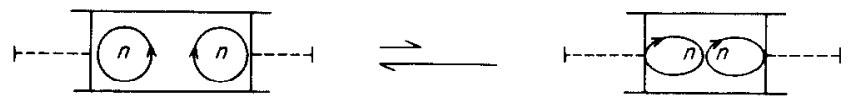

(a)
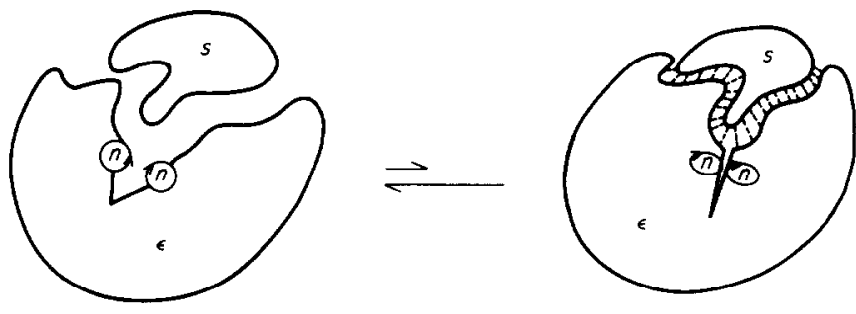

(b)

$$
\begin{aligned}
& n=\text { nuclear charge } \\
& \epsilon=\text { enzyme } \\
& s=\text { substrate }
\end{aligned}
$$

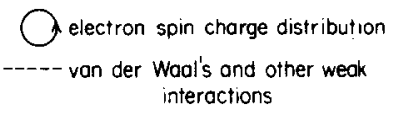

FIG. 1. Pictorial representations of the van der Waal's constraint. The confinement of parallel spin electrons (as in the device illustrated in (a)) increases their momentum fluctuations and favors a phase correlation in their motion (schematically represented in terms of the locations of the arrowheads and distortion of the charge distributions). The non-random character of the phases produces an oscillatory motion of the nuclei and thus the formation of a bound pair can also be thought of in terms of the exchange of quanta associated with these oscillations. Diagram (b) illustrates how the same type of confinement can be imposed as a result of the weak interactions between complementary surfaces of enzyme and substrate.

and are therefore influenced in a coherent way by the nuclear charge densities (roughly, attracted to the same central charge). This establishes a composite system. Alternatively, since the phases are not random, the nuclear wave functions are not independent of the electron wave functions, the nuclei therefore oscillate and consequently the electrons can be thought of as clothed by phonon-like particles (perhaps of quite low energy) and exchanging virtual particles. Other sources of oscillation may be present as well, e.g. short term oscillations resulting from interpenetration and repulsion of the charge clouds and the relatively different time scales of electronic and nuclear motions. Many electrons may be available for potential pairing, thereby increasing the probability of pair formation. In general it would also be expected that the constrained nuclear co-ordinates belong to the enzyme. The coupling energy may be small relative to $k T$, therefore too weak to maintain permanent pairs. 
(B) CONFINEMENT CONSTRAINT AND MOMENTUM FLUCTUATIONS

According to the above, the standard deviation which would be exhibited by measurements on electron position are decreased by the van der Waal's constraint both because of confinement and because of the correlation deriving from exclusion repulsion. According to the uncertainty principle, $\sigma p \sigma x \sim \hbar$, this means that the standard deviation of momentum measurements must increase, implying an increase in the likelihood of both larger and smaller values of the kinetic energy (of the order $\left.\hbar^{2} /\left(2 m_{e}\right)(\sigma x)^{2}\right)$. The inevitability of large values, sometimes given as an explanation for the uncollapsibility of matter, is clearly incompatible with pair formation and pair stability. However, the equally inevitable small values increase the chance of a transient potential energy dominance allowing formation of unstable pairs. It might be noted that this is the converse of the argument for the stability of covalent bonds and resonance structures (since exchange and alternate electronic configurations increase $\sigma x$ and therefore decrease the fluctuations on the kinetic energy). For catalysis, however, the rapid formation of unstable bonds is important and therefore the necessity is for the increased fluctuation which is made possible by confinement and parallel spin.

\section{(c) THERMAL CONSIDERATIONS}

The uncertainty fluctuations discussed above are around an average determined by the temperature. The situation is complicated by the fact that the enzyme (with typically several thousands of atoms) is so highly and flexibly constrained a system that equipartition cannot in general be expected to hold. This actually increases the possibilities for local downward deviations from the average kinetic energy of the environment and also for sources of an attractive interaction. However, to get a worst case idea of the probability of pairing for a small pairing energy $\varepsilon$, consider the equilibrium ratio at constant temperature and pressure given by the formula

$$
n_{p}=n_{u}^{2} \exp \left(\Delta F / N_{0} k T\right),
$$

where $n_{p}$ is the number of pairs, $n_{u}$ is the number of pairable but unpaired electrons, $N_{0}$ is Avogadro's number, $\Delta F=\Delta E-T \Delta S+p \Delta V=F_{u}-F_{p}$, and $\Delta E=N_{0} \varepsilon$. Since what is of interest is a single system, picture $\Delta F$ not as an ensemble average, but rather as an average on a single system taken over a large number of time periods, each just long enough for an ensemble to reach equilibrium. The expected waiting time for the appearance of a first pair $\left(n_{p}=1\right)$ is thus given by

$$
\bar{\tau}=K\langle N\rangle=K\left[\exp \left(-\Delta F / 2 N_{0} k T\right)+2\right] / m,
$$


where $N$ is the number of periods, $m$ is the number of pairable electrons in the system, $2 n_{p}+n_{u}=m N$, and $K$ is a proportionality constant which depends inversely on the rate of equilibration. For pairing to be possible, the potential energy of the paired configuration must be less than that of the unpaired configuration $(\Delta E>0)$. For pairing to be pcrmanent the pairing energy $(\varepsilon)$ must be greater than $k T$. However, if the occurrence of a single pairing event (as opposed to the appearance of a long-lived pair or many contemporaneous pairs) is critical, pairing energies small in comparison to $k T$ are sufficient and allow for a not very large value of $N$ even though pairing is accompanied by an entropy decrease $(\Delta S>0)$. Furthermore, the confinement enhanced fluctuations, the small values of $\varepsilon$, and the absence of any activation barrier once the constraint is imposed allow the pairs to flicker into and out of existence quite rapidly and thus $K$ as well as $N$ can be small and therefore $\bar{\tau}$ quite short.

\section{(D) PAIR FALLING AND ENERGY FLUCTUATIONS}

Paired electrons will differ from other electrons in the system in some quantum number other than spin (and will differ from each other as well, since the exclusion principle must apply within the pair). Thus once the pair is formed there is no barrier preventing it from immediately dropping to a lower level and also delocalizing. This reduces the Coulomb repulsion and also momentum fluctuations which might now break the pair up. It also allows the electrons to escape the coupling interaction which originates from the confinement constraint. In order to break up, however, the pair must reabsorb energy sufficient to re-establish a normal electronic structure. This is because unpairing at the lower levels would conflict with the exclusion principle and thus in effect induces a coupling interaction. This induced interaction could never hold the pair together if energy is available for breaking it. The existence of the pair implies an orbital structure with quantum numbers so unstable that any perturbation resulting from the relcase of energy by falling would always lead to their annihilation and therefore to the reabsorption of at least some of this energy. This can be thought of in terms of the energy-driven entropy decrease inherent in the correlation of electron phases. The reverse process (unpairing) would thus be favored by an entropy driven energy increase. But the only energy available is the energy released by falling (since thermal fluctuation energies are generally too small after falling). The pair may nevertheless persist for a short time as a virtual pair even after it escapes the original coupling interaction, or it may persist if there is some delay in reabsorbing the energy. If the time is very short, the fallen electrons simply cycle back to a normal (possibly rearranged) electronic structure. If it is longer, the pair drops to a lower level 
before cycling back and therefore releases and absorbs more energy. In this case the release of energy may trigger a critical event (such as a nuclear motion) which significantly delays the reabsorption of energy. The pair will be trapped in a low energy state, thereby extending its lifetime over and above that allowed to it as a virtual system.

Let $\tau_{c}$ be the minimum time required for falling to be efficacious (e.g. to initiate a critical event) and denote the pairing energy by $\varepsilon^{\prime}$ (with $\varepsilon^{\prime}<\varepsilon$, because of the decrease in Coulomb repulsion). Pairing is possible if $\sigma \varepsilon^{\prime} \geqslant \varepsilon^{\prime}$. According to the time-energy uncertainty principle $\left(\tau \sim \hbar / \varepsilon^{\prime}\right)$ the pair can thus persist as a virtual pair for an interval of time which increases as $\varepsilon^{\prime}$ decreases, making survival possible for a time interval $\tau_{c} \leqslant \tau$ even under the worst assumptions (i.e. immediate turning off of the original coupling interaction and immediate reabsorption of released energy). Note that the pairing energy uncertainty should be distinguished from violations of energy conservation concomitant to any exchange of virtual particles which mediate the pairing and that, because of the transient nature of the process, pair formation as well as the time for falling can be enhanced by energy fluctuations.

The transient occurrence of parallel spin pairs does not imply instability of orbital structure other than during transitions from one orbital structure to another. It is not inconsistent with arguments against their occurrence in permanent form (in the case of electrons). The confinement argument does not work for antiparallel spins, any attractive interaction would be secondary in this case, and covalent bonding (if possible) would always be favored. Confinement reduces $\sigma x$ for each electron, but is not incompatible with overcoming the Coulomb repulsion, since the mean distance between the electrons is increased by their anticorrelated motion (as illustrated in Fig. 1). In terms of spin and spatial eigenfunctions, confinement initially reduces the difference between the spatial eigenfunctions of two electrons, where the properly antisymmetrized complete eigenfunction consists of an antisymmetric spatial and symmetric spin component. In the energetically favored paired configuration the antisymmetric spatial component is replaced by a distinguishing antisymmetric eigenfunction. Since only one or a few pairs are required to fall, the process is not a Bose condensation. Other configurations, such as triples, though not likely to form, could thus in principle also trigger a critical event.

\section{Role in Enzyme Catalysis and Related Processes}

According to the pairing theory the effectiveness of enzymes is based on the fact that they are macromolecules which undergo cyclic reactions 


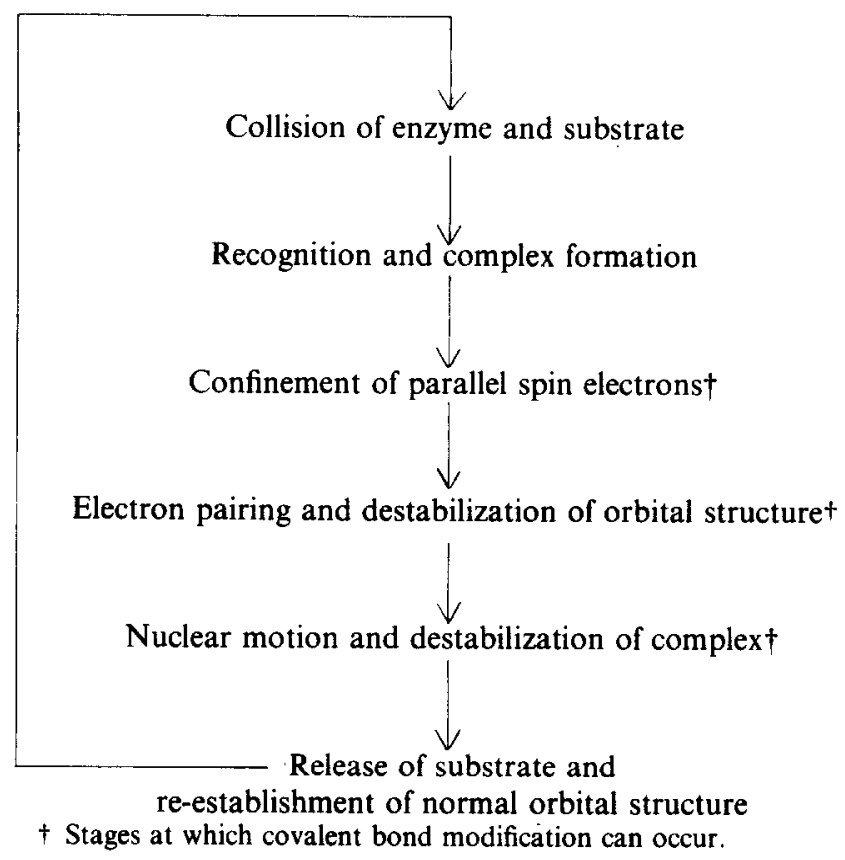

Fig. 2. Sequence of events in enzyme catalysis. The enzyme and substrate are brought together by upward fluctuations to form an activated state. Recognition and complex formation involve a decrease in free energy, which for catalytic processes should not lead to a free energy smaller than that of the product. In the formation process energy dominates entropy and the resulting ordering imposes the confinement constraint. In the presence of downward fluctuations this constraint favors an ordered (paired) state for the confined electrons. The resulting destabilization of the electronic structure results in a transfer of energy to the nuclear configuration, allowing for the dynamic opening of an alternative reaction route and therefore destabilization of the complex (driven by the lower free energy of the uncomplexed system). In a true catalytic process all the transferred energy is released on loan in the sense that it must be recaptured to re-establish the original orbital structure.

involving transient pairing. As summarized in Fig. 2, formation of the enzyme-substrate complex imposes confinement constraints on parallel spin electrons, thereby giving rise to a coupling interaction and also increased downward uncertainty fluctuations in the kinetic energy. Such downward fluctuations (and also downward thermal fluctuations) favor pair formation and the consequent destabilization of the normal orbital structure. There are a number of possible fates for fallen pairs, therefore a number of particular reaction processes. In the one most important for specific catalysis, the energy released when the pair falls and delocalizes is coupled to a nuclear motion (the critical event). The pair is trapped during the motion because 
unpairing is incompatible with the exclusion principle. The resulting induced coupling is highly unstable and thus the pair (or, alternatively, the unstable quantum numbers) will be annihilated as soon as the electrons find an escape route and energy is imparted to them by the fall of the nuclei back to a low energy configuration, thereby allowing for regeneration of the enzyme in its initial form. However, this does not necessarily mean that the complex is regenerated. The major importance of the nuclear motion is that it serves to destabilize the complex if it makes available a low activation energy reaction route which allows (or favors) release of the substrate. Such an active release process makes it possible to support an active recognition process, thereby increasing the speed of complex formation without decreasing the speed of decomposition. Thus the speed of complex formation increases if the complex can assemble itself from any one of a large number of possible initial contacts between enzyme and substrate. But this implies a final complex of lower free energy and in the absence of an active release mechanism would be incompatible with rapid breakup.

Between recognition and release of the substrate a covalent bond must be made or broken. There are three basic types of mechanisms. First, complex formation may increase the chance of covalent bond formation or breakage in an entirely conventional fashion. Bond modification might even precede pairing (whose contribution would then be strictly connected to the motion which destabilizes the complex). The second possibility is that the nuclear motion resulting from pairing causes a nuclear motion of the substrate which facilitates bond formation or breakage. The third possibility is that the paired electrons resurface (with or without triggering a nuclear motion) in an alternate configuration which favors bond formation or breakage for other electrons. Note that pairing cannot directly lead to formation of covalent bonds since the paired electrons have parallel spin and also because the confinement argument would not work in reverse for covalently bound electrons. There are a number of variations of pairing reaction schemes, including variations which are non-cyclic or in which the nuclear motion does not lead to complex breakup. These variations include:

\section{(A) SEQUENTIAL PROCESSES IN CATALYSIS}

Destabilization of one complex may result in the formation of another complex rather than in separation of enzyme and substrate. This allows for further pairing and hence a series of reorientations of the substrate. Such component catalytic processes would lead to release only when a proper end product appears (or when the initial form of the substrate reappears). This also ensures a high degree of selectivity in the bond modification since 
improperly modified substrates would be more likely to undergo a reverse reaction than be released in an improper form.

\section{(B) ALLOSTERIC CONTROL}

A control molecule can be thought of as a substance which causes the enzyme to undergo a nuclear (allosteric) motion to an alternative, active conformation by forming a complex which imposes confinement constraints at a site other than the active site (i.e. at a control site). In general the complex must be sufficiently strong to disfavor release of the control molecule at the end of the process, with the lifetime of the active conformation depending on how much smaller the free energy of the complexed system is than that of the uncomplexed system. The transient pairing mechanism is not necessary for the formation of an enzyme-control molecule complex with an altered shape. However, the energy released by pair falling makes possible a much more radical reorganization of the nuclear configuration than otherwise would be the case.

\section{(C) PERSISTENT MACROMOLECULAR MOTIONS}

If destabilization of the complex leads to a new complex which is the same as the original one, either immediately or through a cycle of processes, the result will be a recurrent change in molecular shape or orientation. The complex in this case need not involve weak interactions between two separate components. Instead the confinement constraints could be imposed by the folding of a single macromolecule.

\section{(D) SELF-ASSEMBLY AND FOLDING}

Highly specific self-assembly of quaternary structures can be facilitated by transient pairing if the process proceeds in stages and each stage results from a highly ordered motion arising from pairing at the previous stage. Selfdisassembly of quaternary structures may also be facilitated if alteration of the milieu results in the imposition of confinement constraints which lead to the disappearance of barriers to complex decomposition. The possibility may also be considered that the folding of individual proteins is self-catalyzed in the sense of proceeding in stages, with confinement constraints imposed by folding at one stage producing directed motions which lead into the next stage.

\section{(E) ENERGY TRANSFER}

If the energy released by pair falling is not recaptured in the reestablishment of a normal orbital structure, the macromolecule cannot be 
the linear inhomogeneous system has two roots

$$
\begin{gathered}
e=\frac{G_{D} \Delta D-D_{D} \Delta G}{\left(\bar{G} D_{D}-\bar{D} G_{D}\right) \Delta t} \\
r=\frac{\Delta \bar{D} \bar{G}-\Delta G \bar{D}}{\left(\bar{G} D_{D}-\bar{D} G_{D}\right) \Delta t}
\end{gathered}
$$

It is assumed (1) that average gastric and duodenal counts represent virtually net counts of the volumes refluxed and emptied and (2) that $e$ and $r$ do not change during a short interval. Furthermore, no changes of the contours of the stomach and the duodenum should occur, and no superpositions of the lower parts of the duodenum and the stomach and of other organs (e.g. gall bladder, liver) with both regions of interest are allowed.

The same equations apply to the measurement of flow rates with a gastric and a duodenal tube after a liquid meal. Total quantities of markers inside the stomach and the duodenum (i.e. volume $\times$ concentration) are substituted for total counts over the regions of interest. Duodenal volume could be measured by intraduodenal marker dilution according to George's (1968) method. Assuming that (i) total amount of duodenal marker remains constant at steady state conditions $\left(V_{D} D_{D}=\right.$ constant) and (ii) reflux of gastric marker is negligible compared with its intragastric contents $\left(r V_{D} G_{D}<e \bar{V} \bar{G}\right)$ the same technique as cited above for measuring flow rates applies with an intragastric tube only. Equations (11) and (12) transform into ( $G$ and $D$ referring to concentrations)

$$
\begin{aligned}
& e=\frac{V_{1} G_{1}-V_{2} G_{2}}{\bar{V} \bar{G} \Delta t} \\
& r=\frac{V_{2} D_{2}-V_{1} D_{1}+e \bar{V} \bar{D} \Delta t}{V_{D} D_{D} \Delta t}
\end{aligned}
$$

For $\Delta t \rightarrow 0$ these two equations change into

$$
\begin{aligned}
& \frac{\mathrm{d}(V G)}{\mathrm{d} t}=-e(V G) \\
& \frac{\mathrm{d}(V D)}{\mathrm{d} t}=-e(V D)+r(V D)_{D}
\end{aligned}
$$

Two ordinary differential equations of the same kind have been suggested for simultaneous measurement of gastric emptying and gastric secretion (Blum, Hegglin, Krejs, Largiadèr, Säuberli \& Schmid, 1976; Dubois, Van Eerdewegh, Line, Van Maele \& Gardner, 1975). 
the confinement constraint gives rise to both the coupling interaction and increased downward fluctuations and in part because the possibility of an energy loan derives from the exclusion principle's role in building up the normal electronic structure. It is of some interest to note that the exclusion principle can also make contributions to catalysis which are more primitive than those associated with transient pairing. Confinement constraints can increase the reactivity of specific electrons, with the degree of confinement influencing the probability that fluctuations fall within the range of momenta which favor the reaction. Confinement enhanced fluctuations may also serve to reduce the lifetime of a complex or be strong enough to trigger a nuclear motion. Thus one can imagine evolutionary selection for confinement on a number of grounds, eventually leading to situations in which pairing becomes more probable and in which the enzyme (or other macromolecule) effectively utilizes the energy released as a consequence of the pairing.

\section{Physical Predictions}

Two novel features of the model are the parallel spin pairing and the contribution of downward fluctuations. Both have physical consequences which are possibly testable. Parallel spin pairing implies that a magnetic moment should mark the pair. This moment should thus appear at the beginning of any nuclear motion, but might in general be short-lived and difficult to detect inside the milieu of the enzyme. The fact that downward fluctuations in average kinetic energy favor pairing implies that the rate of pair formation increases with a decrease in temperature, in contradiction to the usual temperature dependence of reaction rates. The rate of all other aspects of the catalytic process (such as formation of the complex or conventional bond modification) should increase with temperature (excluding temperature increases which lead to denaturation). Very low temperatures will thus stop enzymatic reactions by stopping the conventional chemical mechanisms, but increasing temperatures do not necessarily speed up the overall process because of their slowing influence on pair formation. Components of the reaction with such an anomolous temperature dependence should be marked by the magnetic moment. According to the transient pairing model it is this anomaly which is basically responsible for the departure of enzymes from the classical Arrhenius dependence of rate on temperature.

The model also makes a critical prediction about free energy diagrams for enzymatic reactions. Due to its instability, the real free energy of the complex (e.g. as determined by heat measurements) should be smaller than the free energy as determined from its equilibrium concentration or by kinetic 
methods. In effect, lowering the barriers to complex decomposition creates an apparent free energy which is higher than the real free energy. This is discussed further in the next section.

\section{The Energy Loan Concept and Consistency with Thermodynamics}

The basic feature of any transient pairing scheme is that the energy released by falling and delocalization of pairs gives rise to an orbital structure which is so unstable that transitions leading to its annihilation become the principal energy absorbing transitions. If all the released energy is reabsorbed and the protein (or other macromolecule) returns to its original orbital structure, it is a catalyst in the strict sense (provided that the energy is used for barrier removal). If some of the energy escapes or if the original orbital structure is not re-established, the molecule is a reactant (or is said to be poisoned if it is an enzyme). In either case the energy released and reabsorbed is released on loan and for a strict enzyme all of the energy is released on loan. This energy loan cannot be used to perform macroscopic work since this would clearly violate basic thermodynamic principles. However, this does not prevent it from being used to eliminate potential barriers between free energy minima, thereby allowing switching of substrate molecules (or self-switching) from one minimum to another.

The energy loan concept is illustrated diagrammatically in Fig. 3 and a number of variations (inhibition, perpetual switching, stepwise assembly, sequential catalysis) are illustrated in Fig. 4 . The presence of the enzyme lowers the free energy barrier and the enzyme-substrate complex appears as a new minimum in between the two original minima. Classically, upward fluctuation energies allow the system to escape from one valley to another and thus any deepening of the minima associated with the complex slows the reaction. However, such deepening not only allows increased specificity and speed of complex formation (because it allows for more ways of falling into a specific fit), but also makes possible the imposition of stronger confinement constraints, thus increasing the likelihood of pairing (both because of the resulting coupling interaction and the increasing likelihood of downward fluctuations). The energy released by falling and delocalization is coupled to a motion of the entire complex which dynamically opens up a new reaction route. This can be thought of in terms of a distortion of the potential surface which shortens (and in the limit eliminates) the potential barriers. The situation is represented by the two solid curves in the energy loan diagram (Fig. 3). The upper, normal curve applies to the system with pairable, but unpaired electrons and the lower (short-lived) curve to the system with paired electrons. The arrows on the lower curve indicate that it can only be 


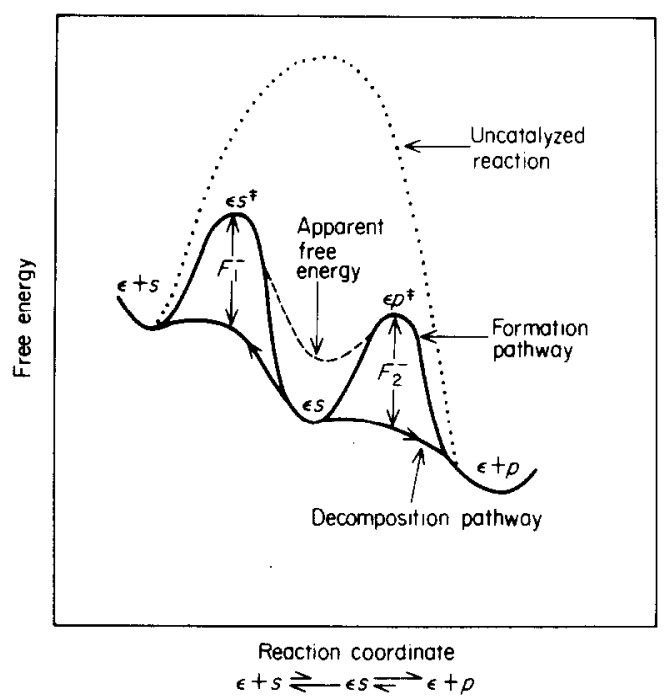

Fig. 3. Schematic energy loan diagram. $\varepsilon$ is the enzyme, $s$ the substrate, $p$ the product, $\varepsilon s^{\ddagger}$ and $\varepsilon p^{\ddagger}$ are activated states, and $\varepsilon s$ is the enzyme substrate complex. $F_{1}^{-}$and $F_{2}^{-}$are energy loans. According to the energy loan model, the instability of the complex allows its actual free energy to be lower than its apparent free energy (i.e. the free energy determined on the basis of its equilibrium concentration or on the basis of the kinetics of the reaction). This is possible because the complex must form by passing through one of the activated states, $\varepsilon s^{\ddagger}$, or $\varepsilon p^{\ddagger}$, but can decompose along the lower decomposition pathway. No violation ol macroscopic thermodynamics results provided that the activation energy advantages are the same for both barriers $\left(F_{1}^{-}=F_{2}^{-}\right)$. The arrows on the lower path represent the fact that it is not possible to enter the path from the uncomplexed configurations without going through the activated states (because the confinement constraint cannot be imposed without complex formation). This irreversibility affects the equilibrium concentration of the complex (giving rise to the apparent free energy), but not the relative equilibrium concentrations of substrate and product. The dotted curve represents the activation barrier for the uncatalyzed reaction.

entered by first forming the complex, not that it cannot be traversed in both directions once the electrons are paired. This is because the confinement constraint is based on complex formation. Once the complex forms (by passing through one of the activated states) electron pairing provides the energy loan which opens up the low activation barrier pathway, giving rise to an activation energy advantage of $F_{1}^{-}$for peak 1 and $F_{2}^{-}$for peak 2 (which may be more or less than the energy loan itself). In effect the dynamic motion of the complex (made possible by the energy loan) allows it to assume a form in which barriers to its decomposition disappear or become very small, thus forcing the system back into one of its original minima. In these minima enzyme and reactant are separated systems and as soon as a normal 

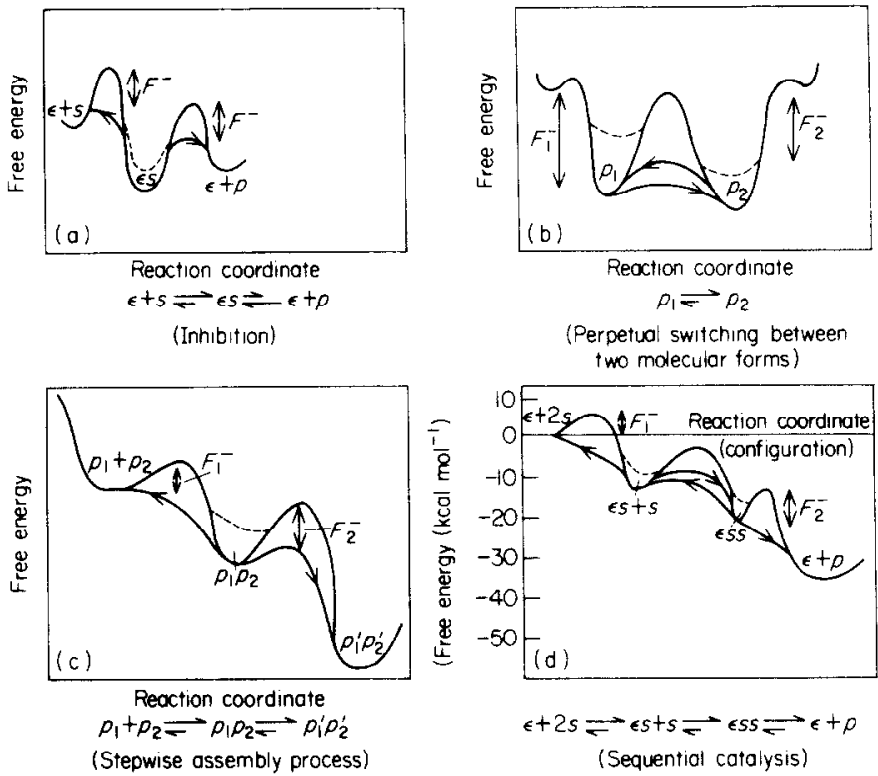

Fic. 4. Schematic energy loan diagrams for a number of macromolecular processes. The components of each diagram have the same interpretation as in Fig. 3. In (a) the free energy of the complexed configuration is lower than that of the uncomplexed configuration. Even with maximum possible barrier shortening, the complexed state is favored and thus $s$ is an inhibitor. (b) is a diagram for a macromolecule which persistently switches between two different confermations. Note that the lower pathways are not necessarily bidirectional and the width of the barrier indicates that a significant change in the reaction co-ordinate is possible. (c) illustrates a stepwise assembly process. Note that the energy loans are not repaid and that the diagram could also be drawn with a negative $F_{1}^{-}$. (d) is a sequential catalysis. The upper curve is adapted from a free energy diagram for catalase $(\varepsilon)$ and $2 \mathrm{H}_{2} \mathrm{O}_{2}(2 s)$ in Setlow \& Pollard (1962, by permission). Note that the activation energy advantages $F_{1}^{-}$and $F_{2}^{-}$are drawn in only once for each pair of decomposition pathways. Even if they are small the rate advantage is significant because of the exponential dependence of reaction rate on the height of the activation barrier.

electronic structure is re-established the barriers to complex formation are reestablished as well. The dashed line in the diagram (between the two peaks) runs through the free energy, $\hat{F}_{\varepsilon s}$, calculated from the formula

$$
[\varepsilon][s] /[\varepsilon s]=\exp \left[\hat{F}_{\varepsilon s}-F_{(\varepsilon+s)}\right] / N_{0} k T,
$$

where $F_{(\varepsilon+s)}$ is the free energy of the enzyme and substrate in the scparated configuration. $\hat{F}_{\varepsilon s}$ is the apparent free energy since it is calculated under the assumption of a single potential curve and therefore of a stable complex.

The energy loan diagram is entirely consistent with macroscopic thermodynamics provided that $F_{1}^{-}=F_{2}^{-}=F^{-}$. To verify this consider the 
various activation energies in Fig. 3. The activation energies for the formation (upper) pathways are given by

$$
\begin{aligned}
& \Delta F_{f 1}^{\ddagger}=F_{\varepsilon s^{\ddagger}}-F_{(\varepsilon+s)} \\
& \Delta F_{f 2}^{\ddagger}=F_{\varepsilon p^{\ddagger}}-F_{(\varepsilon+p)}
\end{aligned}
$$

and the decomposition (lower) pathway by

$$
\begin{aligned}
& \Delta F_{d 1}^{\ddagger}=F_{\varepsilon s^{\ddagger}}-F_{(\varepsilon+s)}-F_{1}^{-} \\
& \Delta F_{d 2}^{\ddagger}=F_{\varepsilon p^{\ddagger}}-F_{(\varepsilon+p)}-F_{2}^{-},
\end{aligned}
$$

where $F_{e s^{\ddagger}}$ and $F_{\varepsilon p^{\ddagger}}$ are the free energies of the activated states. Taking the rate constants as given by $k=A \exp \left(-\Delta F^{\ddagger} / N_{0} k T\right)$,

$$
\begin{aligned}
& A_{f 1} k(\varepsilon s \stackrel{d}{\rightarrow} \varepsilon+s)=A_{d 1} \mathrm{e}^{F_{1}^{-}} k(\varepsilon s \stackrel{f}{\rightarrow} \varepsilon+s) \\
& A_{f^{2}} k(\varepsilon s \stackrel{d}{\rightarrow} \varepsilon+p)=A_{d 2} \mathrm{e}^{F_{3}^{-}} k(\varepsilon s \stackrel{f}{\rightarrow} \varepsilon+p),
\end{aligned}
$$

where the $A_{h i}$ are proportionality constants and $k(\stackrel{f}{\rightarrow} v)$ and $k(u \stackrel{d}{\rightarrow} v)$ are the rate constants for decomposition using the formation and decomposition pathways from $u$ to $v$. According to the theory of absolute reaction rates the $A_{h i}$ can all be taken as $k T / h$ (since translation is the major mode of motion of the activated state). Thus if $F_{1}^{-}=F_{2}^{-}$,

$$
\frac{k(\varepsilon s \stackrel{d}{\rightarrow} \varepsilon+s)}{k(\varepsilon s \stackrel{d}{\rightarrow} \varepsilon+p)}=\frac{k(\varepsilon s \stackrel{f}{\rightarrow} \varepsilon+s)}{k(\varepsilon s \stackrel{f}{\rightarrow} \varepsilon+p)} .
$$

This means the rates for the decomposition pathways are faster, but if the activation energy advantages are equal the relative rates in both direction are the same, implying that the switch from one pathway to the other does not allow the enzyme to affect the final equilibrium (other than the equilibrium concentration of the complex).

For enzymatic processes the symmetry of $F_{1}^{-}$and $F_{2}^{-}$is clearly a thermodynamic requirement. However, it is also reasonable on mechanistic grounds since the weak bonding between reactant and enzyme is in general the same whether or not a covalent bond has been formed or broken, implying that the barriers to complex breakup describe the same physical situation. They should therefore be shortened equally, although of course they could only appear the same if the mechanism of decomposition is the same. The same considerations apply to the symmetry of the proportionality constants, totally apart from any special arguments from absolute reaction rate theory (viz. to the requirement that $A_{f 1} / A_{f 2}=A_{d 1} / A_{d 2}$ ). Also note that the motion of the enzyme should not be thought of as disrupting the weak 
bonds holding the complex together or as ejecting the substrate. If this were the case the enzyme could do work on the environment and thus would not return to its original configuration. Rather it is the dynamic disappearance of the intermediary minimum which ensures that the complex breaks up. The energy released is thus a bona fide energy loan. It derives from falling through the normal energy floor of the complex, is used to suppress an activation barrier, and is then returned to re-establish the floor and hence the barrier. The free energy of the entire system thus does not change as a result of falling-what is lost in terms of electronic energies is gained in terms of configurational or other forms of energy. This is why the falling process must be represented in terms of a change in the potential surface in the energy loan diagram and not in terms of a shift in the position of the enzyme or reactants.

\section{Necessity of an Energy Loan in Thermodynamically Consistent Models of Enzyme Catalysis}

Is it possible to develop a model of enzyme catalysis and related molecular pattern recognition and switching processes which is consistent with thermodynamics but in which the energy loan plays no role? The problem is that high specificity means close fitting between enzyme and appropriate substrate, hence one or more intermediary free energy minima associated with the complex (as in Fig. 3). In order for the reaction to be fast the activation barriers for both complex formation and complex breakup must be small, but at the same time large enough to allow for specificity. For a static potential surface there are two basic possibilities. The first is that there are a small number of allowable close contacts and that these can only be made with selected substrates. In this case the intermediary minimum will be quite shallow (i.e. the free energy of binding not overly large relative to $k T$ ) and the complex capable of breaking up rapidly, but the likelihood of actually making these contacts is small and therefore the rate of formation slow. The second possibility is that any of a large number of initial contacts between enzyme and substrate allow the complex to assemble itself through a series of stages each of lower free energy, thus lowering the activation barrier to complex formation. However, this necessarily deepens the minimum associated with the complex, slowing its breakup. This conflict cannot be eliminated by supposing a multidimensional reaction surface with the property that at any point in the reaction pathway the barriers are always small in some direction, for any such low barrier pathway could always be traversed in the reverse direction and thus would obviate barriers altogether (in contradiction to the above argument). The implication is that the potential surface must be non-static. Given the requirement for cyclicity of 


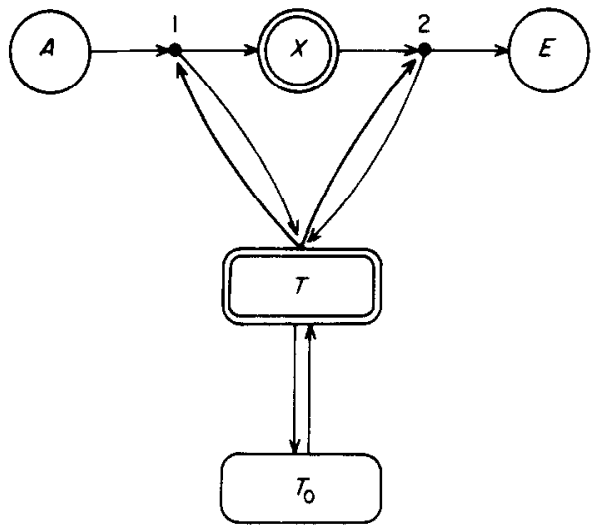

FIG. 1. Schematic representation of the model exothermic reaction. A diagram (A) $\rightarrow \rightarrow(B)$ represents a reaction $A \rightarrow B$. Generation or transfer of heat is also indicated by an arrow, and thick arrows represents the effects of thermal feedback. The meanings of other symbols are given in the text.

The positive feedback effect of the heat of reaction appears through the dependence of reaction coefficients $K_{1}$ and $K_{2}$ on the system temperature $T$ as given by Arrhenius' formula

$$
\begin{aligned}
& K_{1}(T)=k_{1} \exp \left(-\varepsilon_{1} / \mathrm{k}_{B} T\right), \\
& K_{2}(T)=k_{2} \exp \left(-\varepsilon_{2} / k_{B} T\right) .
\end{aligned}
$$

These are indicated by thick arrows in Fig. $1 . k_{B}$ is the Boltzmann constant.

Using these reaction parameters one may write down a set of kinetic equations as follows,

$$
\begin{aligned}
\frac{\mathrm{d} X}{\mathrm{~d} t} & =K_{1} A-K_{2} X, \\
C & \begin{array}{l}
\mathrm{d} T \\
\mathrm{~d} t
\end{array}=Q_{1} K_{1} A+Q_{2} K_{2} X-\frac{l S}{V}\left(T-T_{0}\right),
\end{aligned}
$$

where $C$ is the heat capacity of the system per unit volume, $l$ is the thermal conductivity, $S / V$ is the surface to volume ratio of the system at heat bath contact, respectively. Introducing appropriate scale constants these equations are transformed into a simpler form, i.e.

$$
\begin{aligned}
& \frac{\mathrm{d} x}{\mathrm{~d} s}=\Phi(\theta)-\kappa x \Phi(\theta / \varepsilon), \\
& \frac{\mathrm{d} \theta}{\mathrm{d} s}=a \Phi(\theta)+a \eta \kappa x \Phi(\theta / \varepsilon)-\sigma\left(\theta-\theta_{0}\right),
\end{aligned}
$$


The energy loan is completely compatible with thermodynamics. Fluctuations are of course violations of thermodynamics on a microscopic scale, with upward fluctuations tantamount to conversion of heat into small scale work and downward fluctuations tantamount to a local cooling. This is true both in the classical theory and in the present theory, except that downward fluctuations play no role in the former. In both cases fluctuations only determine the rates at which macroscopic states are transformed into one another. In neither case do they determine the energies or entropies of these states and therefore in no case can they provide energy for macroscopic work. This is also true for the energy loan made possible by downward fluctuation and transient pairing, provided that this loan is completely repaid. From the standpoint of the present theory an enzyme can thus be considered as a macromolecule which makes possible a cyclic reaction involving an energy loan which is used for barrier removal and which arises from transient pairing. The loan either opens a pathway which allows switching of specific substrate from one form to another or it may allow self-switching of the macromolecule between two states. If the loan is not fully repaid the energy released can perform work (e.g. by affecting equilibria) but the reaction cannot be cyclic and the molecule involved therefore is not an enzyme. It may, however, contribute to a specific energy transfer.

The energy loan very much amplifies the pattern recognition and switching capabilities of proteins and other macromolecules and can be expected to occur in any thermodynamically consistent model of enzyme catalysis which achieves both high specificity and speed (because of the requirement of a non-static potential surface). This is true not only for enzymes proper, but also for related fundamental processes involving specificity and ordered nuclear motion (e.g. allosteric control, membrane processes, channeled transport, self-assembly, persistent molecular shape changes or reorientations). In each such process the free energy of the complex as defined by its equilibrium (the apparent free energy) should differ from the real free energy. This is why speed and specificity are both possible. Each such process should also exhibit the anomalous temperature dependence and magnetic moment predicted by the theory.

\section{REFERENCES}

Bardeen, J. CoOper, L. N. \& Schrieffer, J. R. (1957). Phy's. Rev. 108, 1175.

CONRAD, M. (1978). Biophys. J. 21, 21a.

FröHLICH. H. (1968). Physics Lett. 26A, 402.

LitTle, W. A. (1964). Phys. Rev. 134, A 1416

LONDCN, F. (1950). Superfluids, Vol. 1. New York: John Wiley \& Sons. 
Pattee, H. H. (1968). In Towards a Theoretical Biology (C. H. Waddington, ed.), Vol. 1 pp. 67-93. Edinhurgh: University of Edinhurgh Press.

SCHRöDrnger, E. (1944). What Is Life? Cambridge: Cambridge University Press.

Setlow, R. B. \& PollaRd, E. C. (1964). Molecular Biophysics, p. 250. Reading, Mass. Addison-Wesiey.

Singer, S. J. \& Nicolson, G. L. (1972). Science 175, 720. 